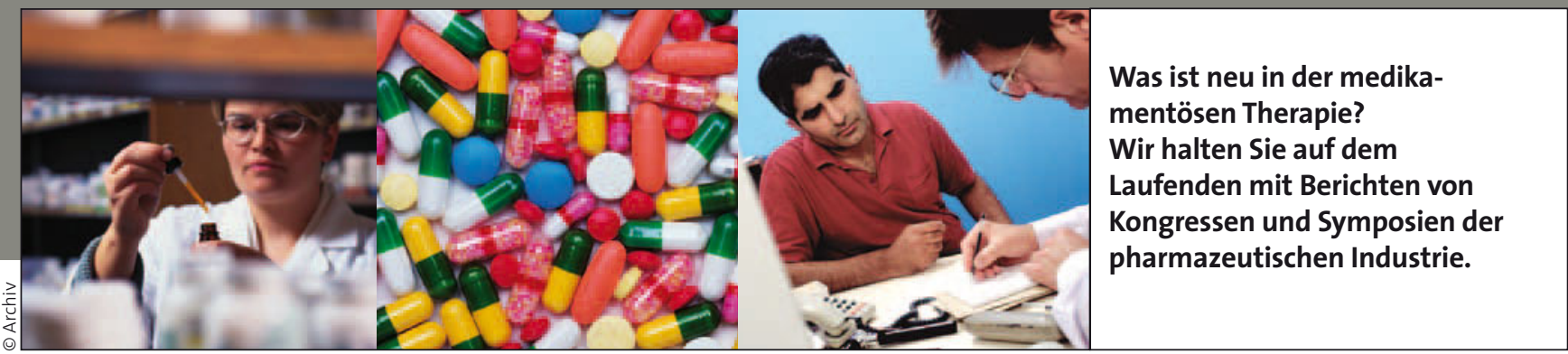

\title{
Bei Sinusitis und Bronchitis
}

\section{Myrtol setzt Sekretolyse in Gang}

- Die akute Form einer Sinusitis wird meist durch den Verschluss des Nebenhöhlen-Ostiums verursacht. Als Folge kommt es zu Störungen von Ventilation und Drainage sowie zur Sekretstagnation, so Prof. Hans Behrbohm, Berlin. Dadurch werden Keimwachstum und Entzündungsreaktion begünstigt. Typische Symptome sind nasale Obstruktion, Kopf-

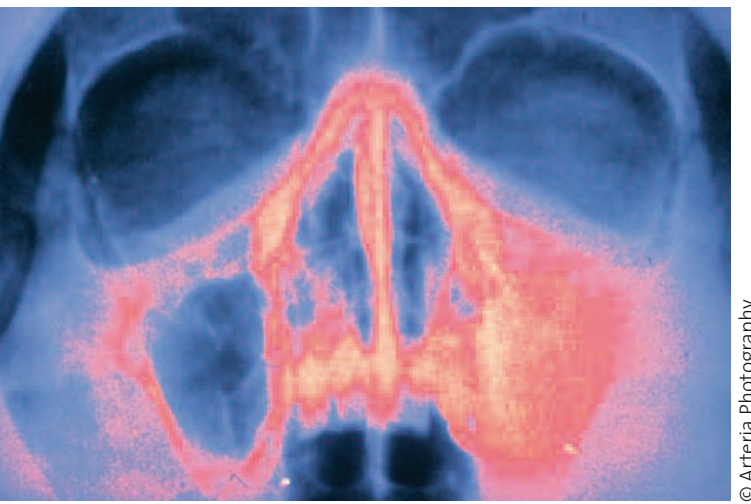

Sekretspiegel bei akuter Sinusitis. und Gesichtsschmerz (v. a. beim Bücken), Hyposmie und nasale Sekretion.

Bei chronisch entzündlicher Schleimhauterkrankungen kommt es zu Veränderungen des Mukoziliarapparates: Sekretorische Becherzellen nehmen ab, die Drüsen bilden vermehrt mukösen statt serösen Schleim, und der Zilienapparat degeneriert. Bei einer Sinusitis kann es zum sog. Etagenwechsel mit Beteiligung der Bronchien kommen.

\section{Klinische Studien belegen den Nutzen von Myrtol}

Auf die Bedeutung von Myrtol standardisiert (GeloMyrtol ${ }^{\circledR}$ ) bei Sinusitis und Bronchitis wies Dr. Thorsten Zehlicke, Hamburg, hin. Dass Präparat mit seinen ätherischen Ölen wirkt in erster Linie sekretolytisch, sekretomotorisch und mukolytisch. Zusätzlich sind antimikrobiellantiinflammatorische, antioxidative und bonchospasmolytische Effekte bekannt.
Die Wirkungen von Myrtol standardisiert sind in zahlreichen experimentellen und klinischen Studien dokumentiert. Zehlicke machte auf eine Good-ClinicalPractice-konforme randomisierte Studie aufmerksam, in der 331 Sinusitispatienten mit dem Myrtolpräparat oder Placebo behandelt wurden. Im Therapieverlauf war eine signifikante Verbesserung der Kernsymptome Kopfschmerz, Schmerz beim Bücken und Druckschmerz über den Nervenaustrittspunkten nachweisbar.

In einer anderen Studie wurden 676 Bronchitispatienten entweder mit Myrtol standardisiert, Cefuroxim, Ambroxol oder Placebo therapiert. Das Myrtolpräparat zeigte nach einer Woche mit 95\% die höchste Ansprechrate und war vergleichbar effektiv wie die anderen Substanzen.

- Dr. med. Andreas Fischer

Quelle: Pressekonferenz „State of the art der Therapie der Rhinosinusitis“, München,

8. Oktober 2010 (Veranstalter: Pohl-Boskamp)

\section{Myokardinfarkt mit Herzinsuffizienz-Zeichen}

\section{Frühe Aldosteronblockade für ein längeres Leben}

- Die Chancen stehen heute gut, die Akutphase eines Myokardinfarkts zu überleben. Dennoch verbleibt ein hohes Restrisiko, nur wenige Wochen oder Monate später zu versterben. Dies gilt vor allem für Infarktpatienten, die klinische Zeichen der Herzschwäche zeigen. Bei ihnen lohnt es sich, umgehend die Therapie mit dem selektiven Aldosteronblocker Eplerenon (Inspra ${ }^{\circledR}$ ) einzuleiten, betonte
Prof. Carsten Tschöpe, Berlin. Denn je früher nach dem akuten Infarkt der Aldosteronspiegel gesenkt wird, desto höher ist die Chance, das prognostisch ungünstige myokardiale Remodelling zu bremsen.

Den Nutzen des frühen Therapiebeginns belegte eine Subgruppenanalyse der Studie EPHESUS: Patienten, bei denen die Therapie zwischen Tag drei und Tag sieben begann, hatten eine noch bessere
Prognose als die Patienten, bei denen erst zwischen Tag acht und Tag 14 mit der Behandlung begonnen wurde. An der EPHESUS-Studie hatten 6632 Infarktpatienten mit klinischen Zeichen der Herzinsuffizienz in den ersten Tagen nach Myokardinfarkt teilgenommen. Die placebokontrollierte Studie belegte, dass die Überlebenswahrscheinlichkeit sehr viel höher ist, wenn Eplerenon zusätzlich zur üb- 